\title{
Sur une inégalité de Garabedian-Shiffer-Nehari pour les fonctions univalentes bornées
}

\author{
par A. Rost et J. Śladkowska (Gliwice)
}

\section{À la mémoire de Franciszek Leja}

Résumé. Soit $F_{T}, 0<T<1$, la famille des fonctions holomorphes et univalentes dans le cercle unité $U=\{z:|z|<1\}$, de la forme

$$
f(z)=b_{1} z+b_{2} z^{2}+\ldots
$$

remplissant les conditions

$$
\begin{gathered}
|f(z)|<1 \quad \text { pour } z \in U, \\
b_{1} \geqslant T .
\end{gathered}
$$

Soit $S_{1}$ la famille des fonctions holomorphes et univalentes dans $U$, de la forme (1), remplissant uniquement la condition (2).

Soit enfin $S\left(b_{1}\right)$ la sous-classe de $S_{1}$ où $b_{1}$ est fixé.

Posons pour $f \in F_{T}, 0<r<1, d=f\left(r e^{i \varphi}\right)$,

$$
F(z)=\sqrt{\frac{d-f(z)}{1-\bar{d} f(z)}} .
$$

$F(z)$ désigne ici une branche de la racine carrée, arbitraire mais fixée, univoque dans $U_{1}$ $=\{z:|z|<r\}$. Pour $(z, \zeta) \in U_{1} \times U_{1}$ les fonctions

$$
\begin{gathered}
A(z, \zeta)=\log \frac{F(z)-F(\zeta)}{(F(z)+F(\zeta))(z-\zeta)}=\sum_{m, n=0}^{\infty} a_{m n} z^{m} \zeta^{n}, \\
B(z, \zeta)=\log \frac{1+F(z) \overline{F(\zeta)}}{1-F(z) \overline{F(\zeta)}}=\sum_{m, n=0}^{\infty} b_{m n} z^{m} \zeta^{n}
\end{gathered}
$$

sont des fonctions holomorphes des variables $z, \zeta$ et $z, \bar{\zeta}$ respectivement.

Soit $H\left(U_{1}\right)$ l'espace linéaire-topologique des fonctions holomorphes dans $U_{1}$ avec la convergence presque uniforme, $H^{\prime}\left(U_{1}\right)$ l'espace conjugué de $H\left(U_{1}\right)$ et $L \in H^{\prime}\left(U_{1}\right)$.

Définissons pour $f \in S_{1}$ la fonctionnelle

$$
\Phi(J)=\operatorname{Re}\left\{L^{2}(A(z, \zeta))+\mid L^{2}(B(z, \zeta))\right\},
$$

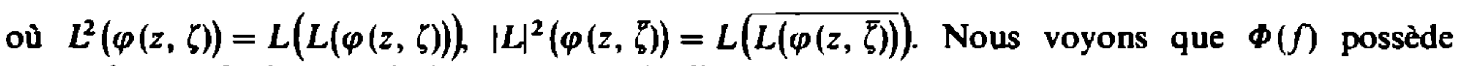
pour chaque $f \in S_{1}$ une dérivée au sens de Fréchet. 
Posons enfin

$$
\begin{aligned}
& \alpha(z, \zeta)=\log \frac{1-x(z+\zeta)+z \zeta-w(z) w(\zeta)}{(z-\zeta) \sqrt{x^{2}-1}}, \\
& \beta(z, \bar{\zeta})=\log \frac{w(z) \overline{w(\zeta)}+x(1+z \bar{\zeta})-\bar{\zeta}-z}{(1-z \bar{\zeta}) \sqrt{x^{2}-1}},
\end{aligned}
$$

,où $w(z)=\sqrt{1-2 x z+z^{2}}, x=\frac{1}{2}(r+1 / r)$.

THÉOREME 1. Soit $f \in F_{T}$ et $d=f\left(r e^{i \varphi}\right)$. Si $L \in H^{\prime}\left(U_{1}\right), \operatorname{Im} L(1)=0$, et si la fonctionnelle $\Phi(f)$, définie par la formule (6), possède une dérivée ne s'annulant en aucun point de la famille $F_{T}$, alors

$$
\Phi(f) \leqslant \operatorname{Re}\left\{L^{2}\left(\alpha\left(z e^{-i \varphi}, \zeta e^{-i \varphi}\right)\right)+|L|^{2}\left(\beta\left(z e^{-i \varphi}, \bar{\zeta} e^{i \varphi}\right)\right)\right\} .
$$

Linégalité (7) est exacte et la fonction extrémale a la forme

$$
f(z)=T z+\ldots
$$

et satisjait à l'équation fonctionnelle

$$
L(A(z, \zeta))+\overline{L(B(z, \bar{\zeta}))}+L(i \varphi)=L\left(\alpha\left(z e^{-i \varphi}, \zeta e^{-i \varphi}\right)\right)+\overline{L\left(\beta\left(z e^{-i \varphi}, \overline{\bar{\zeta}} e^{i \varphi}\right)\right)}
$$

THÉOREMe 2. Dans chaque classe $S\left(b_{1}\right)$ l'inégalité (7) est remplie et elle est exacte dans cette classe. La fonction extrémale satisfait à l'équation (8).

Soit $F_{T}, 0<T<1$, une famille de fonctions holomorphes et univalentes dans le cercle unité $U=\{z:|z|<1\}$, de la forme

$$
f(z)=b_{1} z+b_{2} z^{2}+\ldots
$$

remplissant les conditions

$$
\begin{gathered}
|f(z)|<1 \quad \text { pour } z \in U, \\
b_{1} \geqslant \dot{T} .
\end{gathered}
$$

Ces familles ont été introduites par $\mathrm{Z}$. Charzyński dans [1]. Si nous désignons par $S_{1}$ la classe des fonctions holomorphes et univalentes dans $U$, de la forme (1) et remplissant uniquement la condition (2), en vertu du fait que pour les fonctions de la classe $S_{1}$ nécessairement $0<b_{1} \leqslant 1$, nous avons

$$
S_{1}=\underset{0<T<1}{\bigcup} F_{T} .
$$

Les familles $F_{T}$, contrairement à la famille $S_{1}$, sont compactes. Il en résulte, entre autres, qu'une fonctionnelle arbitraire, réelle et continue, définie dans $F_{T}$, atteint ses valeurs extrémales, ce qu'on ne peut pas dire de la famille $S_{1}$.

En 1953 Z. Nehari a déjà donné dans [7] les conditions nécessaires et suffisantes pour qu'une fonction, qui a un développement de la forme (1) dans le cercle $U$, appartienne a la classe $S_{1}$. Ces conditions ont la forme d'inégalités dans lesquelles, en plus des paramètres arbitraires figurent les coefficients de la fonction $f$. Puisqu'elles sont pareilles aux conditions de Grunsky pour les fonctions de la classe $S$ (famille des fonctions holomorphes 
et univalentes dans le cercle $U$, remplissant les conditions $f(0)=f^{\prime}(0)-1$ $=0$ ), on les a appelées conditions de Grunsky-Nehari. En 1957, [3], Z. Charzyński et H. Śmiałkówna ont trouvé des conditions analogues. En 1969 Z. Nehari a généralisé ces conditions, en y introduisant un paramètre complexe $d, d \notin f(U), d \in U$. Puisqu'en 1967 Garabedian et Schiffer [6] ont donné une pareille généralisation pour la classe $S$, nous allons les appeler conditions de Garabedian -Schiffer-Nehari, en abrégé conditions ou inégalités G-S-N.

En 1970 et 1971, dans deux travaux successifs [4] et [5], W. D. De Temple a trouvé à l'aide d'une méthode variationnelle une nouvelle démonstration du résultat de Nehari. Il a encore élargi le domaine de variabilité du paramètre $d$, en admettant non seulement les valeurs que la fonction n'atteint pas, mais aussi celles qu'elle atteint et les racines d'une certaine équation algébrique.

Les inégalités G-S-N sont exactes dans la famille $S_{1}$ (non pas dans le sens, que De Temple a interprété ce fait, à tort d'après nous). On ne sait pas, en revanche, si elles sont exactes dans les sous-classes compactes $S\left(b_{1}\right)$ sous-classes de $S_{1}$, où $b_{1}$ est fixé. Pour le constater De Temple a créé une technique [5] (the technique of a penalty function), qui lui permet de trouver les fonctions extrémales dans la classe $S\left(b_{1}\right)$ pour certaines simples formes de la fonctionnelle $\mathrm{G}-\mathrm{S}-\mathrm{N}$. Nous entendons la fonctionnelle $\mathrm{G}-\mathrm{S}-\mathrm{N}$ comme le second membre de l'inégalité G-S-N. Toutefois, cette méthode n'a pas d'application dans le cas général.

Les classes $S\left(b_{1}\right)$ ont une relation évidente avec les classes $F_{T}$, notamment $S\left(b_{1}\right) \subset F_{b_{1}}$. En $1952 \mathrm{Z}$. Charzyński a obtenu, dans le travail cité ci-dessus, une variation dans la classe $F_{T}$, à l'aide de laquelle il a obtenu l'équation différentielle-fonctionnelle pour les fonctions extrémales dans cette classe par rapport aux fonctionnelles dépendant d'une quantité finie de coefficients. Dans un travail commun avec W. Janowski, [2], cette équation a été généralisée pour les fonctionnelles arbitraires qui sont différentiables au sens de Fréchet.

Dans cette note on obtient que l'inégalité $\mathbf{G}-\mathbf{S}-\mathbf{N}$ est exacte dans les classes $S\left(b_{1}\right)$. Ça il résulte du fait que cette inégalité est exacte dans les classes $F_{b_{1}}$ et du fait que les fonctions extremales dans $F_{b_{1}}$ appartiennent à $S_{b_{1}}$. Le fait que l'inégalité $\mathrm{G}-\mathrm{S}-\mathrm{N}$ est exacte dans $F_{T}$ résulte de l'application de l'équation sus-mentionnée pour les fonctions extrémales à une fonctionnelle $\mathbf{G}-\mathrm{S}-\mathrm{N}$.

1. Soit $f \in F_{T}, 0<r<1, d=f(r)$. Posons

$$
F(z)=\sqrt{\frac{d-f(z)}{1-\bar{d} f(z)}} .
$$

$F(z)$ signifie ici une branche de la racine carrée, arbitraire mais fixée, 
univoque dans $U_{1}=\{z:|z|<r\}$. Remarquons de plus que pour $(z, \zeta) \in$ $U_{1} \times U_{1}$ les fonctions

$$
\begin{gathered}
A(z, \zeta)=\log \frac{F(z)-F(\zeta)}{(F(z)+F(\zeta))(z-\zeta)}=\sum_{m, n=0}^{\infty} a_{m n} z^{m} \zeta^{n}, \\
B(z, \bar{\zeta})=\log \frac{1+F(z) \overline{F(\zeta)}}{1-F(z) \overline{F(\zeta)}}=\sum_{m, n=0}^{\infty} b_{m n} z^{m} \bar{\zeta}^{n}
\end{gathered}
$$

sont des fonctions holomorphes des variables $z, \zeta$ et $z, \bar{\zeta}$ respectivement; seuls les coefficients $a_{00}, b_{00}$ ne sont pas déterminées d'une façon unique.

Soit $H\left(U_{1}\right)$ l'espace linéaire-topologique, sur le corps des nombres complexes, des fonctions holomorphes dans $U_{1}$ avec la convergence presque uniforme dans $U_{1}$ et $H_{R}^{\prime}\left(U_{1}\right)$ ou $H_{C}^{\prime}\left(U_{1}\right)$ l'espace conjugué de $H\left(U_{1}\right)$ des fonctionnelles linéaires, continues, réelles ou complexes et soit $L \in H_{c}^{\prime}\left(U_{1}\right)$, $\operatorname{Im} L(1)=0$. Du théorème de Caccioppoli sur la représentation générale de la fonctionnelle linéaire et continue dans l'espace des fonctions holomorphes, alors sur la forme générale de l'élément de l'espace $H_{c}^{\prime}\left(U_{1}\right)$, [9], il résulte qu'il existe une fonction $g$ holomorphe dans le domaine $\left\{z:|z|>r^{\prime}\right\}$, où $0<r^{\prime}<r$, telle que pour la fonction arbitraire $f \in H\left(U_{1}\right)$ nous avons

$$
L(f)=\frac{1}{2 \pi i} \int_{|z|=e} f(z) g(z) d z
$$

où $\varrho, r^{\prime}<\varrho<r$, est arbitraire. Si donc $\varphi(z, \zeta)$ est une fonction holomorphe des variables $(z, \zeta) \in U_{1} \times U_{1}, L(\varphi(z, \zeta))$ ( $z$ est une variable simulée, $\zeta$ est un paramètre) est une fonction holomorphe de la variable $\zeta \in U_{1}$, alors la fonctionnelle $L^{2}(\varphi(z, \zeta))=L(L(\varphi(z, \zeta)))$ est bien définie. Il en est de même de la fonctionnelle $|L|^{2}(\psi(z, \bar{\zeta}))=L(\overline{L(\psi(z, \bar{\zeta}))})$ pour la fonction $\psi(z, \bar{\zeta})$ holomorphe comme fonction des variables $(z, \zeta) \in U_{1} \times U_{1}$.

Posons pour $f \in S_{1}$

$$
\Phi(f)=\operatorname{Re}\left\{L^{2}\left(\log \frac{F(z)-F(\zeta)}{(F(z)+F(\zeta))(z-\zeta)}\right)+|L|^{2}\left(\log \frac{1+F(z) \overline{F(\zeta)}}{1-F(z) \overline{F(\zeta)}}\right)\right\}
$$

Remarquons que ni le choix de la branche de la racine dans la définition de $F(z)$, ni celui de la branche du logarithme pour déterminer les fonctions $A(z, \zeta)$ et $B(z, \bar{\zeta})$ n'influe sur la valeur de la fonctionnelle (8).

Nous allons maintenant prouver que $\Phi(f)$ possède pour chaque fonction $f \in S_{1}$ une dérivée au sens de Fréchet, c'est-à-dire, que pour chaque fonction $f \in S_{1}$ il existe une fonctionnelle $A \in H_{R}^{\prime}(U)$, telle que pour chaque nombre $n$, naturel, fixé et pour chaque fonction $h \in H(U)$, telle que $f+h \in S_{1}$, une convergence $h \rightarrow 0$ entraîne que

$$
\frac{|\Phi(f+h)-\Phi(f)-\Lambda(h)|}{\|h\|_{n}} \rightarrow 0,
$$

où $\|h\|_{n}=\sup _{|z|<1-1 / n}|h(z)|$. 
En fait, un calcul facile permet de constater que la fonctionnelle (9) $\quad \Lambda(h)=\operatorname{Re}\left\{L^{2}\left(-h(r) \frac{(1-\bar{d} f(z))(1-\bar{d} f(\zeta))}{(1-|d|)^{2} \eta(z) \eta(\zeta)}-\overline{h(r)} \frac{(d-f(z))(d-f(\zeta))}{\left(1-|d|^{2}\right) \eta(z) \eta(\zeta)}+\right.\right.$

$$
\begin{gathered}
\left.+\frac{1}{(f(z)-f(\zeta)) \eta(z) \eta(\zeta)}\left(\eta^{2}(\zeta) h(z)-\eta^{2}(z) h(\zeta)\right)\right)+ \\
+|L|^{2}\left(h(r) \frac{(d-\overline{f(\zeta)})(1-\overline{d f(z))}}{\left(1-|d|^{2}\right) \eta(z) \overline{\eta(\zeta)}}+\overline{h(r)} \frac{(d-f(z))(1-d \overline{f(\zeta))}}{\left(1-|d|^{2}\right) \eta(z) \overline{\eta(\zeta)}}-\right. \\
\left.-\frac{1}{(1-f(z) \overline{f(\zeta)}) \eta(z) \overline{\eta(\zeta)}}\left(\overline{\left(\eta^{2}(\zeta)\right.} h(z)+\eta^{2}(z) \overline{h(\zeta))}\right)\right\}
\end{gathered}
$$

où

$$
\eta(z)=\sqrt{(d-f(z))(1-\bar{d} f(z))} .
$$

Nous allons calculer les fonctionnelles

$$
D\left(h_{1}\right)=\Lambda\left(h_{1}\right)-i \Lambda\left(h_{1} i\right), \quad \hat{D}\left(h_{2}\right)=\overline{D\left(h_{2}\right)}=\Lambda\left(\hat{h}_{2}\right)+i \Lambda\left(\hat{h}_{2} i\right),
$$

où $h_{1}, h_{2} \in H(U)$ et $\hat{h}_{2}(z)=\overline{h_{2}(\bar{z})}$. Nous obtenons

$$
\begin{aligned}
& D\left(h_{1}\right)=\frac{-h_{1}(r)}{1-|d|^{2}}\left(L\left(\frac{1-\bar{d} f(z)}{\eta(z)}\right)-\overline{L\left(\frac{d-f(z)}{\eta(z)}\right)}\right)^{2}+ \\
& +L^{2}\left(\frac{1}{(f(z)-f(\zeta))(z-\zeta)} \cdot\left(\eta^{2}(\zeta) h_{1}(z)-\eta^{2}(z) h_{1}(\zeta)\right)\right)- \\
& -|L|^{2}\left(\frac{\eta^{2}(z) \overline{h_{1}(\zeta)}}{(1-f(z) \overline{f(\zeta)}) \eta(z) \overline{\eta(\zeta)}}\right)-|L|^{2}\left(\frac{\overline{\eta^{2}(\zeta)} h_{1}(z)}{(1-f(z) \overline{f(\zeta)}) \eta(z) \overline{\eta(\zeta)}}\right) \\
& \hat{D}\left(h_{2}\right)=\overline{\frac{-h_{2}(r)}{1-|d|^{2}}}\left(\overline{L\left(\frac{1-\bar{d} f(z)}{\eta(z)}\right)}-L\left(\frac{d-f(z)}{\eta(z)}\right)\right)^{2}+ \\
& +\overline{L^{2}\left(\frac{1}{(f(z)-f(\zeta)) \eta(z) \eta(\zeta)}\left(\eta^{2}(\zeta) \hat{h}_{2}(z)-\eta^{2}(z) \hat{h}_{2}(\zeta)\right)\right)}- \\
& \left.-|L|^{2}\left(\frac{\overline{\eta^{2}(\zeta)} \hat{h}_{2}(z)}{(1-f(z) \overline{f(\zeta)}) \eta(z)}\right)-\overline{\eta(\zeta)}\right) \overline{|L|^{2}\left(\frac{\eta^{2}(z) \hat{h}_{2}(\zeta)}{(1-f(z) \overline{f(\zeta)}) \eta(z) \overline{\eta(\zeta)}}\right)} .
\end{aligned}
$$

Posons maintenant

$$
\psi(\omega, \lambda)=\omega \frac{1+\omega \lambda}{1-\omega \lambda}
$$


et soit

$$
h_{1}(z)=\psi(f(z), 1 / w), \quad h_{2}(z)=\psi(\hat{f}(z), w)
$$

Alors

$$
h_{1}(z)=f(z) \frac{w+f(z)}{w-f(z)}, \quad h_{2}(z)=f(z) \frac{1+f(z) \bar{w}}{1-f(z) \bar{w}} .
$$

Ces deux fonctions sont holomorphes dans le cercle unité pour $w=e^{i y}, 0 \leqslant y$ $<2 \pi$.

\section{Posons}

$$
\mathfrak{M}(w)=D\left(h_{1}\right)+\hat{D}\left(h_{2}\right)-2 \mathfrak{P}
$$

où

$$
2 \mathfrak{P}=\underset{|w|=1}{\operatorname{Min}}\left(D\left(h_{1}\right)+\hat{D}\left(h_{2}\right)\right)
$$

En vertu de (12), (11) et (10) nous obtenons

$$
\begin{aligned}
& \mathfrak{M}(w)+2 \mathfrak{P}=-\frac{d}{1-|d|^{2}} \frac{w+d}{w-d}\left(L\left(\frac{1-\bar{d} f(z)}{\eta(z)}\right)-\overline{\left(\frac{d-f(z)}{\eta(z)}\right)}\right)^{2}+ \\
& +L^{2}\left(\frac{1}{(f(z)-f(\zeta)) \eta(z) \eta(\zeta)}\left(\eta^{2}(\zeta) f(z) \frac{1+f(z) \bar{w}}{1-f(z) \bar{w}}-\eta^{2}(z) f(\zeta) \frac{w+f(\zeta)}{w-f(\zeta)}\right)\right)- \\
& -|L|^{2}\left(\frac{1}{(1-f(z) \overline{f(\zeta)) \eta(z) \eta(\zeta)}} \overline{\eta^{2}(\zeta)} f(z) \frac{w+f(z)}{w-f(z)}\right)-
\end{aligned}
$$

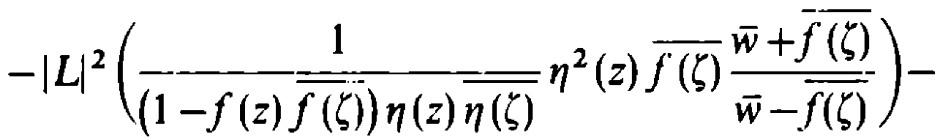

$$
\begin{aligned}
& \left.-\frac{\bar{d}}{1-|d|^{2}} \frac{1+\bar{d} w}{1-\bar{d} w} \overline{\left(L \frac{1-\bar{d} f(z)}{\eta(z)}\right)}-L\left(\frac{d-f(z)}{\eta(z)}\right)\right)^{2}+
\end{aligned}
$$

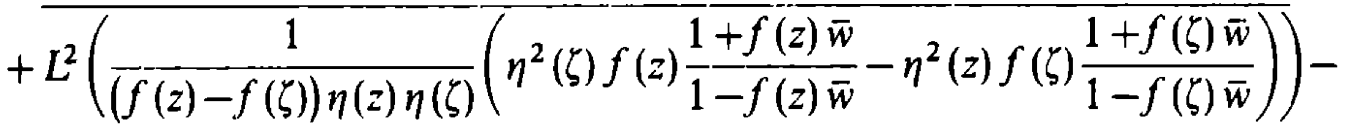

$$
\begin{aligned}
& -|L|^{2}\left(\frac{1}{(1-f(z) \overline{f(\zeta)}) \eta(z) \overline{\eta(\zeta)}} \overline{\eta^{2}(\zeta)} f(z) \frac{1+f}{1-f(z) \bar{w}}\right)-
\end{aligned}
$$

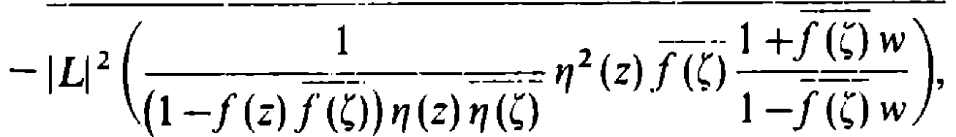

ce qui donne finalement après un calcul assez penible

$$
\mathfrak{M}(w)+2 \mathfrak{P}=\frac{-2 w^{2}}{(w-d)(1-\overline{d w})}\left(L\left(-\frac{\eta(z)}{w-f(z)}\right)-L\left(\frac{\eta(z)}{1-\bar{w} f(z)}\right)\right)^{2},
$$


où

$$
\begin{aligned}
2 \mathfrak{P} & =\operatorname{Min}_{|w|=1} \frac{-2 w^{2}}{(w-d)(1-\overline{d w})}\left(L\left(\frac{\eta(z)}{w-f(z)}\right)-L\left(\frac{\eta(z)}{1-\bar{w} f(z)}\right)\right)^{2} \\
& =\underset{|w|=1}{\operatorname{Min}} \frac{8}{\left|1-e^{-i y} d\right|^{2}}\left(\operatorname{Im} L\left(\frac{\eta(z) e^{-\frac{1}{2} i y}}{1-e^{-i y} f(z)}\right)\right)^{2} .
\end{aligned}
$$

Nous allons prouver que

$$
\mathfrak{P}=0 \text {. }
$$

Dans ce but posons

$$
\varphi(y)=\operatorname{Im} L\left(\frac{\eta(z) e^{-\frac{1}{2} i y}}{1-e^{-i y} f(z)}\right) .
$$

$\varphi$ est une fonction continue et périodique de période $4 \pi$. Comme en outre $\varphi(y+2 \pi)=-\varphi(y)$, cette fonction a dans l'intervalle $\langle 0,2 \pi)$ au moins un zéro, d'où nous obtenons (14).

Posons successivement dans (10)

$$
h_{1}(z)=\psi(z, 1 / u) f^{\prime}(z), \quad h_{2}(z)=\psi(z, u) \hat{f}^{\prime}(z)
$$

ou

$$
\begin{gathered}
h_{1}(z)=z f^{\prime}(z) \frac{u+z}{z-z}, \quad h_{2}(z)=z \overline{f^{\prime}(\bar{z})} \frac{1+z u}{1-z u}, \\
h_{2}(z)=z f^{\prime}(z) \frac{1+\bar{u} z}{1-\bar{u} z} .
\end{gathered}
$$

Ces fonctions sont holomorphes dans le cercle unité pour $u=e^{i x}$, $0 \leqslant x \leqslant 2 \pi$; soit

$$
\mathfrak{N}(u)=D\left(h_{1}\right)+\hat{D}\left(h_{2}\right)-2 \mathfrak{B} .
$$

Alors en vertu de (15), (14) et (10),

$$
\begin{aligned}
\mathfrak{M}(u)= & D\left(z f^{\prime}(z) \frac{u+z}{u-z}\right)+\overline{D\left(z f^{\prime}(z) \frac{1+\bar{u} z}{1-\bar{u} z}\right)} \\
= & -r f^{\prime}(r) \frac{u+r}{u-r} \frac{1}{1-|d|^{2}}\left(L\left(\frac{1-\bar{d} f(z)}{\eta(z)}\right)-L\left(\frac{d-f(z)}{\eta(z)}\right)\right)^{2}+ \\
& +L^{2}\left(\overline{(f(z)-f(\zeta)) \eta(z) \eta(\zeta)}\left(\eta^{2}(\zeta) z f^{\prime}(z) \frac{u+z}{u-z}-\eta^{2}(z) \zeta f^{\prime}(\zeta) \frac{u+\zeta}{u-\zeta}\right)\right)- \\
& -|L|^{2}\left(\overline{\left(1-f(z) \overline{f(\zeta)) \eta(z) \overline{\eta(\zeta)}} \overline{\eta^{2}(\zeta)} z f^{\prime}(z) \frac{(1 / \bar{u})+z}{(1 / \bar{u})-z}\right)-}\right.
\end{aligned}
$$




$$
\begin{aligned}
& -|L|^{2}\left(\frac{1}{(1-f(z) \overline{f(\zeta))}) \eta(z) \overline{\eta(\zeta)}} \eta^{2}(z) \bar{\zeta} \overline{f^{\prime}(\zeta)} \frac{\bar{u}+\bar{\zeta}}{\bar{u}-\bar{\zeta}}\right)- \\
& \left.-\overline{r f^{\prime}(r)} \frac{1+u r}{1-u r} \overline{\left(L\left(\frac{1-\overline{d f(z)}}{\eta(z)}\right)\right.}-L\left(\frac{d-f(z)}{\eta(z)}\right)\right)^{2}+ \\
& +\overline{L^{2}\left(\frac{1}{(f(z)-f(\zeta)) \eta(z) \eta(\zeta)}\left(\eta^{2}(\zeta) z f^{\prime}(z) \frac{1+\bar{u} z}{1-\bar{u} z}-\eta^{2}(z) \zeta f^{\prime}(\zeta) \frac{1+\bar{u} \zeta}{1-\bar{u} \zeta}\right)\right)}- \\
& -|L|^{2}\left(\frac{1}{(1-f(z) \overline{f(\zeta)) \eta(z) \overline{\eta(\zeta)}}} \overline{\eta^{2}(\zeta)} z f^{\prime}(z) \frac{1+\bar{u} z}{1-\bar{u} z}\right)- \\
& -\mid L^{2}\left(\frac{1}{(1-f(z) f(\zeta)) \eta(z) \eta(\bar{\zeta})} \eta^{2}(z) \bar{\zeta} \overline{f^{\prime}(\zeta)} \frac{1+u \bar{\zeta}}{1-u \bar{\zeta}}\right) .
\end{aligned}
$$

En utilisant de nouveau la représentation générale de la fonctionnelle linéaire $L$, nous voyons que $\mathfrak{N}(u)$ est une fonction méromorphe dans l'anneau $P=\{u: \varrho<|u|<1 / \varrho\}, \varrho<r$, et possède des pôles uniquement dans les points $r$ et $1 / r$. De plus, remarquons que la dérivée (9), prise en un point arbitraire $f \in F_{T}$, se prolonge d'une façon continue sur les fonctions méromorphes dans $U$, dont tous les pôles appartiennent à l'ensemble $Q$ $=\{u: \varrho<|u|<1\} \backslash\{r\}$.

En vertu du théorème principal de [2], p. 45, et de la conclusion II, p. 55 , nous constatons que:

Si la dérivée (9) de la fonctionnelle (8) ne s'annule identiquement en aucun point de la famille $F_{T}$, chaque fonction $f \in F_{T}$, qui réalise le maximum de la fonctionnelle (8) dans la famille $F_{T}$, remplit les conditions suivantes: l'équation

(I) Dans l'anneau $P_{1}=\{z: \varrho<|z|<1\}$ la fonction $f$ satisfait à

$$
\left(\frac{z f^{\prime}(z)}{f(z)}\right)^{2} \mathfrak{M}(f(z))=\mathfrak{N}(z),
$$

où $\mathfrak{M l}(w)$ et $\mathfrak{Y}(z)$ sont définies par les formules (12), (15) et (14).

(II) La fonction $\mathfrak{M}(w)$ est méromorphe dans le domaine suité entre les courbes $\quad C_{e}^{\prime}=\left\{w: w=f\left(\varrho e^{i \varphi}\right), \quad 0 \leqslant \varphi \leqslant 2 \pi\right\} \quad$ et $\quad C_{e}^{\prime \prime}=\left\{w: w=1 / f\left(\varrho e^{i \varphi}\right)\right.$, $0 \leqslant \varphi \leqslant 2 \pi$; et elle admet des pôles uniquement aux points $d$ et $1 / \bar{d}$. La fonction $\mathfrak{N}(z)$ est méromorphe dans l'anneau $P$ et elle posède des pôles uniquement aux points $r$ et $1 / r$.

(III) Les fonctions $\mathfrak{D l}(w)$ et $\mathfrak{N}(z)$ atteignent sur $\partial U$ des valeurs qui ne sont pas négatives.

(IV) Le premier coefficient du développement de la fonction $f$ est égal à $T$. 
Il résulte tout de suite des propriétés de la fonction $\mathfrak{N}(z)$ sus-mentionnées, que la fonction $\mathfrak{N}(z)$ peut avoir uniquement des racines de degré pair sur $\partial U$ et le premier membre de l'équation (16) est une fonction méromorphe dans $P_{1}$, elle possède un pôle au point $r$ et elle est le carré de la fonction

$$
\mathfrak{N}_{1}(z)=\sqrt{2} z f^{\prime}(z)\left(L\left(\frac{\eta(\zeta)}{\eta(z)(f(\zeta)-f(z))}\right)+\overline{L\left(\frac{\eta(\zeta)}{\overline{\eta(z)}(1-\overline{f(z)} f(\zeta))}\right)}\right)
$$

en vertu de (13). Dans cet anneau $\mathfrak{N}_{1}(z)$ est une fonction analytique, biunivoque, $r$ est un point de ramification pour $\eta(z)$. Elle se prolonge comme fonction analytique sur l'anneau $P_{2}=\{z: \varrho<|z| \leqslant 1\}$. En fait, si $z^{*} \in \partial U$ et $\mathfrak{R}\left(z^{*}\right) \neq 0$ dans un certain voisinage du point $z^{*}$ il existe deux branches univoques de la racine carrée de $\mathfrak{N}(z)$ et l'une d'elles doit être identique à l'une des deux branches $\mathfrak{N}_{1}(z)$ dans une partie commune de l'anneau $P_{1}$ et de ce voisinage. Si, au contraire $\mathfrak{N}\left(z^{*}\right)=0, \mathfrak{N}(z)=\left(z-z^{*}\right)^{2 k} \mathfrak{N}(z)$ dans un voisinage du point $z^{*}$, où $\widetilde{\mathfrak{N}}(z)$ est une fonction holomorphe et elle n'est pas nulle. Donc dans ce voisinage du point $z^{*}$ il existe deux branches univoques de la racine de $\mathfrak{N}(z)$, de même que précédemment. L'une d'elles est identique à l'une des deux branches de $\mathfrak{N}_{1}(z)$ dans une partie commune de ce voisinage et de lanneau $P_{1}$. Ainsi nous avons démontré que $\mathfrak{N}_{1}(z)$ se prolonge analytiquement sur l'anneau $\boldsymbol{P}_{2}$; elle est, en outre, réelle sur $\partial U$.

Considérons maintenant la fonction

$$
\mathfrak{N}_{2}(z)=\sqrt{\frac{(z-r)(1-r z)}{z}} \mathfrak{N}_{1}(z)
$$

dans l'anneau $P_{2}$ : c'est une fonction biunivoque, elle n'a pas de points de ramification dans l'anneau $P_{1}$ et elle est réelle sur $\partial U$. Posons

$$
\mathfrak{N}_{3}(z)=-L\left(\frac{\sqrt{z} \sqrt{(\zeta-r)(1-r \zeta)}}{\zeta-z}\right)+\overline{L\left(\frac{\sqrt{\bar{z}} \sqrt{(\zeta-r)(1-r \zeta)}}{1-\bar{z} \zeta}\right)}
$$

C'est encore, de même que $\mathfrak{R}_{2}$, une fonction biunivoque dans $\boldsymbol{P}_{2}$, elle n'a pas de points de ramification dans $P_{2}$ et elle est réelle sur $\partial U$. Considérons maintenant la fonction

$$
\begin{aligned}
H(z) & =\frac{1}{\sqrt{2}} \sqrt{\frac{(z-r)(1-r z)}{z}} \mathfrak{N}_{1}(z)+\mathfrak{N}_{3}(z) \\
& =L\left(\frac{\sqrt{z} \sqrt{(z-r)(1-r z)} f^{\prime}(z) \eta(\zeta)}{\eta(z)(f(\zeta)-f(z))}-\frac{\sqrt{z} \sqrt{(\zeta-r)(1-r \zeta)}}{\zeta-z}\right)+
\end{aligned}
$$




$$
\begin{aligned}
+ & L\left(\frac{\sqrt{\bar{z}} \sqrt{(\bar{z}-r)(1-r \bar{z}) f^{\prime}(z) \eta(\zeta)}}{\overline{\eta(z)}(1-\overline{f(z)} f(\zeta))}+\frac{\sqrt{\bar{z}} \sqrt{(\zeta-r)(1-r \zeta)}}{1-\bar{z} \zeta}\right) \\
= & \sqrt{z}\left\{L\left(\frac{\sqrt{(z-r)(1-r z)} f^{\prime}(z) \eta(\zeta)}{\eta(z)(f(\zeta)-f(z))}-\frac{\sqrt{(\zeta-r)(1-r \zeta)}}{\zeta-z}\right)+\right. \\
+ & \left.-L\left(\frac{\sqrt{(\bar{z}-r)(1-r \bar{z})} \overline{f^{\prime}(z)} \eta(\zeta)}{\overline{\eta(z)}(1-\overline{f(z)} f(\zeta))}+\frac{\sqrt{(\zeta-r)(1-r \zeta)}}{1-\bar{z} \zeta}\right)\right\}
\end{aligned}
$$

dans l'anneau $\boldsymbol{P}_{2}$. La fonction entre accolades se prolonge comme fonction univoque et holomorphe sur le cercle $U$, car les fonctions

$$
\frac{\sqrt{(z-r)(1-r z)} f^{\prime}(z) \eta(\zeta)}{\eta(z)(f(\zeta)-f(z))}-\frac{\sqrt{(\zeta-r)(1-r \zeta)}}{\zeta-z}
$$

et

$$
\frac{\sqrt{(\bar{z}-r)(1-r \bar{z})} \overline{f^{\prime}(\bar{z})} \eta(\zeta)}{\overline{\eta(z)}(1-\overline{f(z)} f(\zeta))}+\frac{\sqrt{(\zeta-r)(1-r \zeta)}}{1-\bar{z} \zeta}
$$

sont univoques et holomorphes respectivement par rapport aux variables $\zeta, z$ et $\zeta, \bar{z}$ dans $U_{1} \times U$ est encore une conséquence de la représentation intégrale de la fonctionnelle $L$. Donc la fonction $H(z)$ est analytique, biunivoque et elle possède un point singulier, qui est un point de ramification à l'origine. En outre, elle est réelle sur la circonférence $\partial U . H^{2}(z)$ est déjà une fonction univoque et holomorphe dans $U$, réelle sur $\partial U$, donc d'après le principe de la symétrie de Schwarz elle se prolonge comme fonction holomorphe au plan fermé $C$. Alors $H^{2}(z)=$ const, et puisque $H(0)=0$, $H(z)=0$ pour tout $z \in U$. Ainsi nous avons obtenu la relation suivante pour la fonction extremale:

$$
\begin{aligned}
\frac{f^{\prime}(z)}{\eta(z)}\left[L\left(\frac{\eta(\zeta)}{f(\zeta)-f(z)}\right)+L\left(\frac{\eta(\zeta)}{1-\overline{f(z)} f(\zeta)}\right)\right] & \left.=\overline{\left(\frac{\sqrt{(\zeta-r)(1-r \zeta)}}{\zeta-z}\right)-L\left(\frac{\sqrt{(\zeta-r)(1-r \zeta)}}{1-\bar{z} \zeta}\right)}\right]
\end{aligned}
$$

pour $z \in P_{2}$.

Maintenant notre but est de calculer les valeurs de la fonctionnelle (8) pour cette fonction extrémale.

Pour faciliter les calculs introduisons les notations suivantes:

$$
w(z)=\sqrt{1-2 x z+z^{2}},
$$

où

$$
x=\frac{1}{2}(r+1 / r) .
$$


Alors (18) prendra la forme

(19)

$$
\frac{f^{\prime}(z)}{\eta(z)}\left[L\left(\frac{\eta(\zeta)}{f(\zeta)-f(z)}\right)+\overline{L\left(\frac{\eta(\zeta)}{1-\overline{f(z)} f(\zeta)}\right)}\right]=\frac{1}{w(z)}\left[L\left(\frac{w(\zeta)}{\zeta-z}\right)+\overline{L\left(\frac{w(\zeta)}{1-\bar{z} \zeta}\right)}\right] .
$$

Pour calculer les intégrales de la dernière équation, différentions par rapport à $z$ resp. $\bar{z}$ les fonctions $A(\zeta, z)$ et $B(\zeta, \bar{z})$; Nous obtenons

$$
\begin{gathered}
\frac{\partial A(\zeta, z)}{\partial z}=-\frac{f^{\prime}(z)}{f(\zeta)-f(z)} \frac{\eta(\zeta)}{\eta(z)}+\frac{1}{\zeta-z} \\
\frac{\partial B(\zeta, \bar{z})}{\partial \bar{z}}=-\frac{\overline{f^{\prime}(z)}}{1-\overline{f(z)} f(\zeta)} \frac{\eta(\zeta)}{\overline{\eta(z)}}
\end{gathered}
$$

En posant (20) et (21) dans (19) et en tenant compte du fait que la fonctionnelle $L$ est continue et linéaire:

$$
L\left(\frac{\partial}{\partial z}(\varphi(\zeta, z))\right)=\frac{d}{d z} L(\varphi(\zeta, z)) \quad \text { et } \quad \overline{L\left(\frac{\partial}{\partial \bar{z}}(\varphi(\zeta, \bar{z}))\right)}=\frac{d}{d z} \overline{L(\varphi(\zeta, \bar{z}))}
$$

nous obtenons

(22) $\frac{d}{d z} L(A(\zeta, z))+\frac{d}{d z} \overline{L(B(\zeta, \bar{z}))}=L\left(\frac{1}{\zeta-z}\right)-\frac{1}{w(z)}\left[L\left(\frac{w(\zeta)}{\zeta-z}\right)+\overline{L\left(\frac{w(\zeta)}{1-\bar{z} \zeta}\right)}\right]$

pour $z \in \boldsymbol{P}_{2}$. Posons

$$
\begin{aligned}
& \alpha(\zeta, z)=\log \frac{1-x(z+\zeta)+z \zeta-w(z) w(\zeta)}{(\zeta-z) \sqrt{x^{2}-1}}=\sum_{j, k=0}^{\infty} A_{j k}(x) \zeta^{j} z^{k}, \\
& \beta(\zeta, \bar{z})=\log \frac{\overline{w(z)} w(\zeta)+x(1+\bar{z} \zeta)-\bar{z}-\zeta}{(1-\bar{z} \zeta) \sqrt{x^{2}-1}}=\sum_{j, k=0}^{\infty} B_{j k}(x) \zeta^{j} \bar{z}^{k}
\end{aligned}
$$

où ces développements sont valides dans $U_{1} \times U_{1}$, et où seuls les coefficients $A_{00}(x)$ et $B_{00}(x)$ ne sont pas définis univoquement. Un simple calcul montre que

$$
\frac{\partial \alpha}{\partial z}=\left(1-\frac{w(\zeta)}{w(z)}\right) \frac{1}{\zeta-z}
$$

et

$$
\begin{gathered}
\frac{\partial \beta}{\partial \bar{z}}=-\frac{1}{1-\bar{z} \zeta} \frac{w(\zeta)}{\overline{w(z)}} \\
\frac{\partial}{\partial z} \overline{\beta(\zeta, \bar{z})}=\overline{\left(\frac{\partial \beta(\zeta, \bar{z})}{\partial \bar{z}}\right)}=-\frac{1}{1-\bar{\zeta} z} \overline{\frac{w(\zeta)}{w(z)}}
\end{gathered}
$$


Donc finalement

$$
\left.\left.\frac{d}{d z}[L(A(\zeta, z))+\overline{L(B(\zeta, \bar{z})})\right]=\frac{d}{d z}[L(\alpha(\zeta, z))+\overline{L(\zeta, \bar{z})})\right]
$$

découle de (22), (23) et (24).

Cette égalité est remplie pour chaque $z \in P_{2}$, les fonctions qui figurent dans les deux membres de l'équation (27) possèdent un point unique de ramification dans $r$. Maintenant en calculant les intégrales des deux membres de (27) sur une courbe arbitraire, qui est située dans $P_{2}$ et unit $z \in P_{2}$ avec $r$, il faut d'abord prendre au point $z$ les mêmes valeurs des fonctions multivoques figurant dans les deux membres de l'égalité (27), et ensuite prolonger ces fonctions sur la courbe mentionnée jusqu'à un point arbitraire $z^{\prime} \neq r$, alors nous obtenons

$$
\begin{aligned}
& {[L(A(\zeta, z))+\overline{L(\overline{B(\zeta, \bar{z})})}]-\left[L\left(A\left(\zeta, z^{\prime}\right)\right)+\overline{L\left(B\left(\zeta, \bar{z}^{\prime}\right)\right)}\right]} \\
& =[L(\alpha(\zeta, z))+\overline{L(\beta(\zeta, \bar{z}))}]-\left[L\left(\alpha\left(\zeta, z^{\prime}\right)\right)+\overline{L\left(\beta\left(\zeta, \bar{z}^{\prime}\right)\right)}\right] .
\end{aligned}
$$

Ensuite nous constatons que

$$
\begin{gathered}
A\left(\zeta, z^{\prime}\right) \underset{z^{\prime} \rightarrow r}{\rightarrow}-\log (r-\zeta), \quad B\left(\zeta, \bar{z}^{\prime}\right) \underset{z^{\prime} \rightarrow r}{\rightarrow} \log 1, \\
\alpha\left(\zeta, z^{\prime}\right) \underset{z^{\prime} \rightarrow r}{\rightarrow}-\log (r-\zeta)+\log \frac{1-r^{2}}{2 r \sqrt{x^{2}-1}}=-\log (r-\zeta), \\
\quad \beta\left(\zeta, \bar{z}^{\prime}\right) \underset{z^{\prime} \rightarrow r}{\rightarrow}-\log \frac{1-r^{2}}{2 r \sqrt{x^{2}-1}}=\log 1
\end{gathered}
$$

et, en passant à la limite avec $z^{\prime} \rightarrow r$ dans (28), et en tenant compte du fait que $\operatorname{Im} L(1)=0$, nous obtenons

$$
L(A(\zeta, z))+\overline{L(B(\zeta, z))}=L(\alpha(\zeta, z))+\overline{L(\beta(\zeta, \bar{z}))}+C,
$$

où $C$ est un nombre constant réelle, et dans les deux membres de (29) il existe des fonctions multivoques de la variable $z \in P_{2}$ avec un point de ramification unique dans $r$. Puisque les fonctions $A(\zeta, z), B(\zeta, \bar{z}), \alpha(\zeta, z)$, $\beta(\zeta, \bar{z})$ examinées dans l'ensemble $\{\zeta:|\zeta|=\varrho\} \times P_{2}$ se prolongent comme fonctions analytiques biunivoques des variables $(\zeta, z)$ et $\zeta, \bar{z}$ respectivement sur l'ensemble $U_{1} \times U_{1}$ admettant les développements (6), (7) et (23), (24), les fonctions $L(A(\zeta, z)), L(B(\zeta, \bar{z})), L(\alpha(\zeta, z))$ et $L(\beta(\zeta, \bar{z}))$ sont des fonctions analytiques biunivoques dans $U_{1}$ sans points de ramification et la valeur de la fonctionnelle $\operatorname{Re} L$ pour la fonction qui figure au premier membre de (29), et par ce seul fait au second membre aussi, ne dépend pas du choix des branches respectives; donc nous obtenons l'égalité

$$
\operatorname{Re}\left(L^{2}(A(\zeta, z))+|L|^{2}(B(\zeta, \bar{z}))\right)=\operatorname{Re}\left(L^{2}(\alpha(\zeta, z))+|L|^{2}(\beta(\zeta, \bar{z}))\right)
$$


THÉORÈME 1. Soit $f \in F_{T}$ et $d=f\left(r e^{i \varphi}\right), 0<r<1, \quad x=\frac{1}{2}(r+1 / r)$. Si $L \in H_{C}^{\prime}\left(U_{1}\right), \quad \operatorname{Im} L(1)=0, \Lambda(h)$ définie par la formule (9) ne s'annule identiquement en aucun point de la famille $F_{T}$, et $A(z, \zeta), B(z, \bar{\zeta}), \beta(z, \bar{\zeta})$ et $\alpha(z, \zeta)$ sont définies par les formules (6), (7) et (23), (24), Tinégalité suivante est remplie

$$
\begin{aligned}
\operatorname{Re}\left\{L^{2}(A(z, \zeta))+|L|^{2}(\right. & B(z, \bar{\zeta}))\} \\
& \leqslant \operatorname{Re}\left\{L^{2}\left(\alpha\left(z e^{-i \varphi}, \zeta e^{-i \varphi}\right)\right)+|L|^{2}\left(\beta\left(z e^{-i \varphi}, \bar{\zeta} e^{i \varphi}\right)\right)\right\} .
\end{aligned}
$$

Linégalité (30) est exacte et la fonction extrémale $f$ a la forme

$$
f(z)=T z+\ldots
$$

et satisfait dans $U$ à léquation fonctionnelle

$$
\begin{aligned}
L\left(\log \frac{F(z)-F(\zeta)}{(F(z)+F(\zeta))(z-\zeta)}\right)+ & L\left(\log \frac{1-F(z) \overline{F(\zeta)}}{1+F(z) \overline{F(\zeta)}}\right)+L(i \varphi) \\
& =L\left(\alpha\left(z e^{-i \varphi}, \zeta e^{-i \varphi}\right)\right)+\overline{L\left(\beta\left(z e^{-i \varphi}, \overline{\zeta e^{i \varphi}}\right)\right)} .
\end{aligned}
$$

Démonstration. Dans le cas où $\varphi=0$, ce théorème est une conclusion immédiate des considérations précédentes. Dans le cas où $\varphi \neq 0$ posons

$$
L^{*}(h(z))=L\left(h\left(z e^{-i \varphi}\right)\right), \quad \text { pour } h \in H(U) \quad\left(L^{*}\left(h\left(z e^{i \varphi}\right)\right)=L(h(z))\right) .
$$

De plus, $L^{*} \in H^{\prime}(U)$, possède les mêmes propriétés que la fonctionnelle $L$. Posons encore

$$
f^{*}(z)=e^{-i \varphi} f\left(z e^{i \varphi}\right)
$$

et

$$
d^{*}=f^{*}(r)=e^{-i \varphi} f\left(r e^{i \varphi}\right)=e^{-i \varphi} d .
$$

En tenant compte du fait que $f^{*} \in F_{T}$, en posant

$$
F^{*}(z)=\sqrt{\frac{d^{*}-f^{*}(z)}{1-\bar{d}^{*} f^{*}(z)}}
$$

et en appliquant à la fonctionnelle $L^{*}$ et à la fonction $f^{*}$ l'inégalité (30) et l'égalité (31) pour $\varphi=0$, nous obtenons

$$
\begin{aligned}
& \operatorname{Re}\left\{L^{* 2}\left(\log \frac{F^{*}(z)-F^{*}(\zeta)}{\left(F^{*}(z)+F^{*}(\zeta)\right)(z-\bar{\zeta})}\right)+\left|L^{*}\right|^{2}\left(\log \frac{1+F^{*}(z) \overline{F^{*}(\zeta)}}{1-F^{*}(z) \overline{F^{*}(\zeta)}}\right)\right\} \\
& \leqslant \operatorname{Re}\left\{L^{* 2}(\alpha(z, \zeta))+\left|L^{*}\right|^{2}(\beta(z, \bar{\zeta}))\right\} \\
& L^{*}\left(\log \frac{F^{*}(z)-F^{*}(\zeta)}{\left(F^{*}(z)+F^{*}(\zeta)\right)(z-\zeta)}\right)+L^{*}\left(\log \frac{1-F^{*}(z) \overline{F^{*}(\zeta)}}{1+F^{*}(z) \overline{F^{*}(\zeta)}}\right) \\
& =L^{*}(\alpha(z, \zeta))+\overline{L^{*}(\beta(z, \bar{\zeta}))}
\end{aligned}
$$


Mais en vertu de $(32)-(35)$ et de la condition $\operatorname{Im} L(1)=0$ nous constatons que

$$
\begin{aligned}
\operatorname{Re}\left\{L^{* 2}\left(\log \frac{F^{*}(z)-F^{*}(\zeta)}{\left(F^{*}(z)+F^{*}(\zeta)\right)(z-\zeta)}\right)+\left|L^{*}\right|^{2}\left(\log \frac{1-F^{*}(z) \overline{F^{*}(\zeta)}}{1+F^{*}(z) \overline{F^{*}(\zeta)}}\right)\right\} \\
=\operatorname{Re}\left\{L^{2}\left(\log \frac{F(z)-F(\zeta)}{(F(z)+F(\zeta))(z-\zeta)}\right)+\mid L^{2}\left(\log \frac{1-F(z) \overline{F(\zeta)}}{1+F(z) \overline{F(\zeta)}}\right)\right\}
\end{aligned}
$$

$\left.\operatorname{Re}_{i} L^{* 2}(\alpha(z, \zeta))+\left|L^{*}\right|^{2}(\beta(z, \bar{\zeta}))\right\}=\operatorname{Re}\left\{L^{2}\left(\alpha\left(z e^{-i \varphi}, \zeta e^{-i \varphi}\right)\right)+|L|^{2}\left(\beta\left(z e^{-i \varphi}, \bar{\zeta} e^{i \varphi}\right)\right)\right\}$

et l'inégalité (30) est équivalente à l'inégalité (36), tandis que l'égalité (37) prend la forme (31), ce qui achève la démonstration.

En vertu de (4) l'inégalité (30) est remplie pour chaque fonction de la classe $S_{1}$ et en outre pour chaque $b_{1}, 0<b_{1}<1$, il existe une fonction de classe $S_{1}$ avec le premier coefficient $b_{1}$, pour laquelle l'inégalité devient une égalité.

De cette manière le théorème suivant a été démontré:

ThÉORÈME 2. Dans chaque classe $S\left(b_{1}\right)$ l'inégalité (30) est remplie et elle est exacte dans cette classe. La fonction extrémale satisfait à réquation (31).

Ainsi le problème posé au début est entièrement résolu.

\section{Références}

[1] Z. Charzyński, Sur les fonctions unicalentes bornées, Rozprawy Mat. 2 (1953), 1-57.

[2] -. W. Jannowski, Sur l'éguation générale des fonctions extrémales dans la famille des fonctions uniralentes hornées, Ann. Univ. M. Curie-Sklodowska 4 (7), Sec. A (1950), $41-56$.

[3] -, H. Śmialkówna, Sur certaines conditions nécessaires et suffisantes pour que les fonctions holomorphes dans le cercle unité soient unitalentes et hornées. Bull. Soc. Sci. Lettres, Lódż Sec. III, 8 (2) (1957), 1-7.

[4] D. W. De Temple, On coefficient inequalities for bounded univalent functions, Ann. Acad. Sci. Fenn. Ser. AI, 469 (1970), 1-19.

[5] -, Generalizations of the Grunsky-Nehari inequalities, Arch. Rational Mech. Anal. 44 (1971), 93-120.

[6] P. R. Garabedian, M. Schiffer, The local muximum theorem for coefficients of univalent functions, ibidem 26 (1967), 1-32.

[7] Z. Nehari, Some inequalities in the theory of functions, Trans. Amer. Math. Soc. 75 (1953), 256286.

[8] -, Inequalities for the coefficients of univalent functions, Arch. Rational. Mech. Anal. 34 (1969). 301-330.

[9] G. Schober, Univalent Functions - Selected Topics, Lecture Notes Math. 478. Berlin . Heidelberg New York 1975, 34-38. 